\section{MuSK+ Myasthenia Gravis Presenting with Severe Sleep Apnea, Respiratory Dysfunction and Response to Rituximab}

Monica Alcantara, $\mathrm{MD}^{1}$; Clodagh Ryan, $\mathrm{MD}^{2}$; Nathan Stall, MD ${ }^{3}$; Robert Jackson ${ }^{4}$; Neha Patel, MD ${ }^{4}$; Hans Katzberg, MD ${ }^{1}$

${ }^{1}$ Ellen \& Martin Prosserman Centre for Neuromuscular Diseases, Toronto General Hospital, University Health Network, 5th floor, Room 307. University of Toronto, Toronto, ON Canada, M5G2C4

${ }^{2}$ Toronto General Hospital, University Health

Network, University of Toronto, Toronto, ON Canada, M5G2C4

${ }^{3}$ Mount Sinai Hospital, Division of Internal Medicine, University Health Network, University of Toronto, Suite 475 - 600 University Avenue, Toronto, ON, Canada, M5G 1X5

${ }^{4}$ University of Toronto, Toronto, ON, Canada M5B 1W8

Keywords: Myasthenia gravis, MuSK myasthenia, respiratory, neuromuscular junction, apnea, hypoventilation

\section{Introduction}

MuSK + MG is a rare subtype of MG that affects predominantly female individuals and manifests with striking features of cranial, bulbar and respiratory muscle weakness during the disease course (1). Isolated or predominantly respiratory failure is rare at presentation, however progression to severe bulbar weakness and respiratory crisis are distinctive features of $\mathrm{MuSK}+\mathrm{MG}$, which poses significant diagnostic challenges, considering the low prevalence of the disease (2-4). Furthermore, patients frequently have poor response to standard immunotherapies and higher rate of life-threatening crisis, adding significant challenges for disease management. We report a case of reversible respiratory muscle dysfunction at disease onset, leading to dyspnea, sleep apnea and hypoventilation in a patient with MuSK + MG. While it is acknowledged that the current case is a rare presentation of a rare disease, it raises a number of treatment considerations and as such we wish to highlight these features through this report.

\section{Case Report}

Clinical presentation and initial investigations

A 53-year-old woman presented to the emergency department with dyspnea and orthopnea that progressed over 5 months. Past medical history was relevant for type 2 diabetes ( $\mathrm{HbAlC}$ of $6.4 \%$, without any micro or macrovascular complications), hypertension and depression. She had recently been diagnosed with severe obstructive sleep apnea (OSA). The sleep study done at another facility showed a total apnea-hypopnea index (AHI) of 53.4/hour in NREM and 63.1/hour in REM sleep, along with sustained oxygen desaturation suggestive of hypoventilation (minimum saturation of $55.1 \%$ and $94.8 \%$ time spent below $90 \%$ saturation). On initial neurologic examination, cranial nerves were normal. There was no facial, or bulbar weakness, ptosis or restricted ocular movements and palate elevation was symmetric. Muscle power was mildly reduced in neck extension/flexion, arm abductors and hip flexors (4+/5 MRC - Medical Research Council muscle scale). She had an unremarkable cardiorespiratory examination and was of normal body mass index (BMI).

Nocturnal non-invasive ventilation with bi-level positive airway pressure ST (Resmed Stellar ${ }^{\mathrm{TM}}$ 150) plus supplemental oxygen was started following her admission to hospital. She required an inspiratory positive airway pressure (IPAP) of $21 \mathrm{cmH} 2 \mathrm{O}$, an expiratory positive airway pressure (EPAP) of $9 \mathrm{cmH} 2 \mathrm{O}$ and a backup rate of 16 breaths per minute to abolish the obstructive events and improve gas exchange.

Chest CT was normal, with no mediastinal or pulmonary masses, infiltrative or infectious processes. Diaphragm M mode/two-dimensional B mode ultrasound imaging during quiet inspiration showed normal baseline thickness and a normal thickening fraction of $35 \%$ with inspiration. A transthoracic echocardiogram was normal. Forced vital capacity (FVC) was 1.18 liters seated and 0.69 liters supine (supine: seated FVC difference of $41.5 \%$ ). Venous blood gas measurements showed $\mathrm{pH}$ of 7.38, pCO2 of $60 \mathrm{~mm} \mathrm{Hg}, \mathrm{pO} 2$ of $52 \mathrm{mmHg}$, HCO3 of $36 \mathrm{mmol} / \mathrm{L}$. Phrenic nerve conduction studies were normal. Repetitive nerve stimulation of the facial and accessory nerves showed no significant decrement in amplitude (4\% in the frontalis, $2 \%$ in trapezius). Electromyography of the upper limb
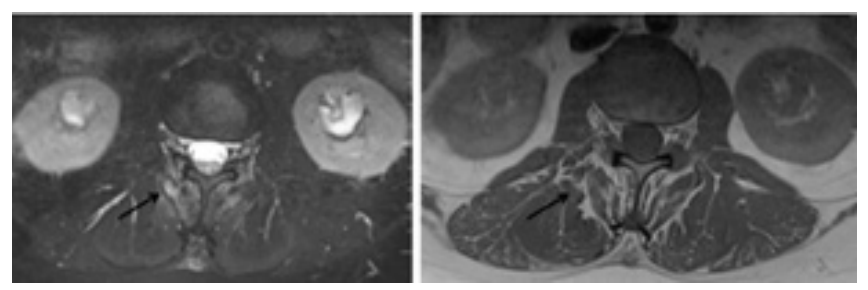

Figure 1. Axial T2 images with fat suppression of the lumbar paraspinal muscles showing atrophy of the intrinsic paraspinal musculature and fatty replacement at L3 spinal level (arrows). 
muscles was normal, but there were myopathic motor unit action potentials and early recruitment in the hip flexors and paraspinal muscles, with fibrillation potentials and complex repetitive discharges. MRI spine showed atrophy and edema of the intrinsic paraspinal musculature, fatty replacement, with normal sign and muscle volumes in bilateral hip and thigh muscles (Figure 1). CK was $39 \mathrm{U} / \mathrm{L}$ (normal <149). A dried blood spot test for acid maltase deficiency was normal.

A latissimus dorsi biopsy was done in the seated position as she could not tolerate prone position due to respiratory distress. A Next Generation Sequencing gene panel including 90 genes encompassing limb-girdle muscular dystrophies, rigid spine, nemaline, myofibrillar and centronuclear myopathies, inclusion myopathies, metabolic myopathies and congenital myasthenic syndromes was also done and did not find any pathogenic gene mutations or variants of unknown significance. Singlefiber EMG of the frontalis muscle was abnormal, showing $56 \%$ abnormal pairs, $44 \%$ blocking and mean elevated jitter at 270.1us. Acetylcholine receptor (AchR), low-density lipoprotein receptor-related protein 4 (LRP4), and AntiMUSK antibodies were requested.

\section{Clinical Progression and Treatment}

Symptoms progressed over the next month with increased dyspnea and orthopnea, new-onset diplopia, mild dysphagia and dysarthria, mild left-sided fatigable ptosis, mild bilateral facial weakness and restricted extraocular movements with diplopia on extreme gaze. The patient developed a constant head drop (neck extension 3/5 $\mathrm{MRC}$ ), and strength was reduced in the hip and shoulder girdle muscles. Myasthenia Gravis Foundation of America (MGFA)(5) class was IVb, as defined as severe weakness predominantly affecting oropharyngeal and respiratory muscles. Myasthenia Gravis Impairment Index (MGII)(6) was 46 out of 84 possible points (higher scores meaning more severe disease) and she was again admitted to the

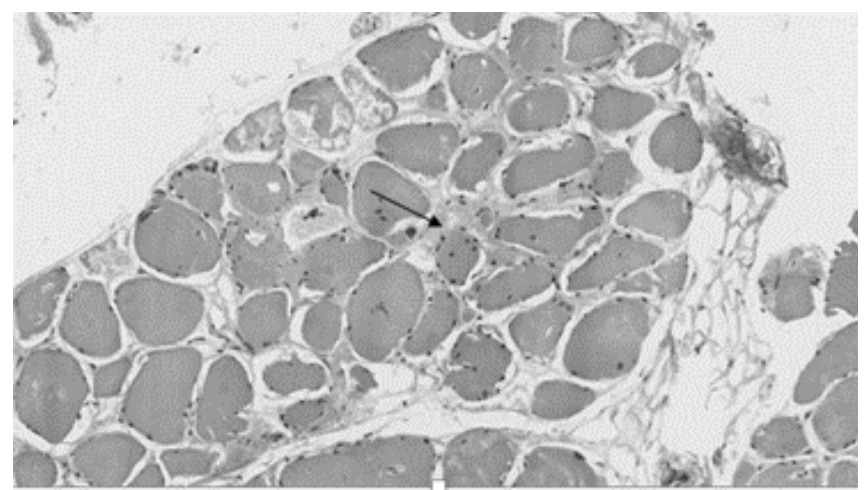

Figure 1. Morphological findings in latissimus dorsi muscle biopsy showing moderate variability in fiber size, scattered atrophic fibers and focal endomysial fibrosis (arrow). hospital to facilitate treatment and for monitoring.

A formal clinical swallowing assessment was not suggestive of pharyngeal propulsion impairment and there were no signs of laryngeal penetration. Muscle biopsy showed focal endomysial fibrosis, fatty infiltration, normal glycogen content and no inflammatory changes (Figure 2). Gomori trichrome did not show any ragged red fibres, nemaline bodies or rimmed vacuoles. NADH showed normal checkerboard pattern without cores. PAS showed normal glycogen content and ORO showed normal lipid content. Congo red did not demonstrate any amyloid deposit. Immunostain for TDP-43 was negative.

Acetylcholine receptor (AchR) and low-density lipoprotein receptor-related protein 4 (LRP4) were negative. Anti-MUSK antibodies were positive, with high titers at $52 \mathrm{nmol} / \mathrm{L}$ (normal $<13 \mathrm{nmol} / \mathrm{L}$ ), confirming the diagnosis of MUSK + MG.

During this hospitalization she received a 5-day course of intravenous immunoglobulin (IVIG, 2g/Kg), and started a course of oral prednisone at a dose of $50 \mathrm{mg} /$ day $(0.6 \mathrm{mg} / \mathrm{Kg} /$ day $)$. This therapy was chosen due to the recent progression of weakness, head drop, respiratory and bulbar symptoms given the likely favorable/fast response in severe sudden clinical deterioration. Plasmapheresis was considered, but given the restricted availability, this treatment was deferred and would be considered in case of further deterioration. Over the following two weeks, she had a significant improvement in diplopia/ptosis, however, no effect on respiratory and bulbar dysfunction, which prohibited safe discharge from hospital.

She was subsequently started on Rituximab infusions (710mg intravenous once a week, corresponding to approximately $375 \mathrm{mg} / \mathrm{m} 2$ ) and after the second infusion she had a significant improvement of her overall strength, shortness of breath and orthopnea; complete resolution of diplopia, ptosis, dysarthria and dysphagia. Given the clinical improvement and to minimize time in hospital, the remaining 2 weekly infusions were performed in an infusion centre, as the first wave of the COVID-19 pandemic accelerated in the Province of Ontario, including outbreaks in the hospital where she was admitted.

One month after the last rituximab infusion, she had a significant improvement of her neck weakness, without any need to support her head (MRC 4) and could ambulate for several blocks with no shortness of breath. There was minimal proximal weakness in her lower limbs $\left(4^{+}\right)$and no fatigability. MGII score was 3 out of possible 64 points on the score questionnaire. MGFA class was now IIIb. Prednisone was progressively tapered to $10 \mathrm{mg}$ over the following 2 months to optimize type 2 diabetes mellitus control. Her IPAP settings were adjusted to $14 \mathrm{~cm} \mathrm{H}_{2} \mathrm{O}$, 
EPAP to $8 \mathrm{cmH} 2 \mathrm{O}$, improved since the initial adjustment, with complete resolution of her sleep apnea symptoms. Arterial blood gas measurements normalized: $\mathrm{pH}$ of 7.42, pCO2 of $31 \mathrm{~mm} \mathrm{Hg}$, pO2 of $104 \mathrm{~mm} \mathrm{Hg}$, HCO3 of 20 $\mathrm{mmol} / \mathrm{L}$. Additionally, at the 3-month mark, there was a significant improvement in FVC (3.3 litres), respiratory pressures were also normal (MIP 60cmH2O, MEP 95cm H2O, SNIP max 91cmH2O). She could lie flat for longer periods and no longer experienced orthopnea.

\section{Discussion}

We report a 53-year-old woman presenting with dyspnea and sleep apnea for 6 months and mild proximal weakness on examination who demonstrated myopathic changes on paraclinical tests and was ultimately diagnosed with MuSK + myasthenia gravis. She exhibited partial response to IVIG and steroids and subsequent near complete response to Rituximab. Although there have been previous reports of $\mathrm{MuSK}+\mathrm{MG}$ producing respiratory dysfunction, myopathic changes and response to Rituximab, our case is unique due to the early presentation with isolated sleep apnea and hypoventilation, followed by mild limb and bulbar weakness, severe neck weakness, the comprehensive clinical neuromuscular characterization, association with markedly high MuSK antibody titres and treatment decisions posed by the rapidly progressing COVID-19 pandemic.

Isolated respiratory dysfunction at presentation has been reported in a minority ( $2 \%$ of patients) in two large cohorts and in smaller case series of patients which included MuSK + MG. (2-4). In spite of the rarity of a respiratory presentation, respiratory crisis (MGFA class $\mathrm{V})$ can occur at some point in the disease course in 32 to $52 \%$ of $\mathrm{MuSK}+\mathrm{MG}$ cases reported in the literature (1, 7). Our patient developed mild limb and bulbar weakness and neck weakness only after presenting with respiratory dysfunction for 6 months, imposing significant challenges for clinical diagnosis and treatment.

In the only previously reported case of $\mathrm{MuSK}+$ MG presenting with severe OSA (AHI of 31/hour), the patient had concurrent bulbar dysfunction as part of their presentation, something not observed in our case initially. An increased prevalence of sleep disordered breathing/ OSA has been reported in some studies including patients who are AChR antibody positive as well as in children with MG $(8,9)$; however, not all studies have found this association (10). In a recent single-center study, the risk of OSA in MG patients was associated with the same risk factors as the overall population, including male sex and obesity (11). Risk factors leading to sleep apnea in MuSK+ MG are lacking in the literature, and notably our patient was neither male nor obese. She also did not have prominent bulbar symptoms until later in the disease course, which has also been implicated as a cause of airway obstruction during sleep leading to apnea. As such, it is difficult to conclusively determine the mechanism leading to OSA in our patient, however, the marked improvement in OSA after immunotherapy implicates $\mathrm{MG}$ as the major reason for this finding.

Our patient did appear to show signs of diaphragmatic dysfunction which was worse in REM sleep and associated with supine changes in FVC of $41.5 \%$, exceeding the $25 \%$ limit, which is usually associated with diaphragmatic weakness (12). In our patient, normal diaphragmatic ultrasound and evidence of myopathic changes in the paraspinal and proximal muscles tested indicates that the diaphragm was neither the sole driver of respiratory dysfunction nor severely damaged or atrophied (13). We could not directly assess whether myopathic changes in respiratorymusclescouldhavecontributed toher respiratory weakness, as we did not perform electromyography of the diaphragm or intercostal muscles. Hemidiaphragm atrophy has been reported in one patient with $\mathrm{MuSK}+\mathrm{MG}$ who presented with isolated dyspnea and had decreased diaphragmatic thickness and excursion with inspiration (12). This is in contrast to our patient with normal thickness and thickening fraction, and is not surprising given the reversible nature of the respiratory deficits.

Chronic myopathic changes including muscle atrophy and fatty replacement on MRI imaging in limb, facial and ocular muscles have been previously reported in patients with MuSK + MG (14, 15). Experimental studies suggest that MuSK plays a significant role in the development and maintenance of the neuromuscular junction, with anterograde and retrograde signaling roles, resulting in AChR clustering in the post-synaptic folds, a process essential for neuromuscular transmission (16). Histologic analysis in $\mathrm{MuSK}+\mathrm{MG}$ involved muscles shows increase in cytochrome c oxidase (COX)-negative fibres, mitochondrial aggregates and myofibrillar disarray, implicating mitochondrial dysfunction as a contributor to pathophysiology $(14,17,18)$. Our patient had edema and fatty replacement of the intrinsic paraspinal muscles, along with chronic myopathic changes, which indicate that part of her weakness might be due to an underlying myopathic process. An extended genetic panel for myopathy was negative, and as such, the possibility of a concomitant hereditary myopathy is highly unlikely. After confirming the diagnosis of MuSK + MG, additional staining and electron microscopy were deferred.

We believe that the clinical presentation in our patient could be related to markedly elevated serum concentrations 
of MuSK Immunoglobulin G4 (IgG4) antibodies, as those levels correlate with clinical changes $(1,19)$. High levels of MuSK antibodies have been associated with severe presentations, such as respiratory failure, as was the case in our patient. To our knowledge this is the highest reported antibody titer $(52 \mathrm{nmol} / \mathrm{L})$ as compared to other cases in the literature $(4,20)$. Also, improvement in response to Rituximab infusions seems to correlate with MuSK antibody titers as was the case in our patient (20). In recent years, there is growing evidence that Rituximab treatment in refractory MG, particularly MuSK + MG leads to significant and sustained improvement and should be considered an early therapeutic option in severe cases $(3,21,22)$.

Although steroids can induce early clinical remission in some MuSK + patients, progressive deterioration often occurs in spite of prolonged, high dose steroids and can result in a fixed steroid-induced myopathy $(1,23,24)$. Our patient experienced improvement in bulbar and respiratory function only after the first Rituximab infusion, in contrast to IVIG and steroid treatment, which only resulted in improvement of the ocular symptoms. Although some patients can have a delayed response to steroid or IVIG treatment, this is uncommon, and in our case, it was felt that the additional time spent in the hospital waiting for a response would confer additional risk of COVID-19 infection. Although the MGFA COVID-19 management recommendations (25) state that other immunomodulatory treatments should be considered prior to Rituximab, we propose this clinical scenario as one example where this treatment should be considered early, and ideally while selfisolating.

In summary, we highlight through this case that respiratory muscle dysfunction leading to dyspnea and hypoventilation can be an isolated presentation of MuSK + MG. Our case further provides evidence that myopathic changes can occur in MuSK + MG and contribute to respiratory symptoms through reversible dysfunction of diaphragm and accessory breathing muscles. Additionally, in patients being considered for neuromuscular causes of respiratory dysfunction including sleep apnea, single fiber EMG and reflexive MG antibody panels, including MuSK testing is necessary to completely exclude patients with MuSK + MG who can present in this manner. Finally, we recommend that early treatment with Rituximab should be considered in bulbar and respiratory presentations of MUSK + MG, as this can cause rapid improvement and avoid complications and ineffectiveness of more traditional immunotherapy.

\section{References}

1. Evoli A, Alboini PE, Damato V, Iorio R, Provenzano C, Bartoccioni E, et al. Myasthenia gravis with antibodies to MuSK: an update. Ann N Y Acad Sci. 2018;1412(1):82-9.

2. Evoli A, Tonali PA, Padua L, Monaco ML, Scuderi F, Batocchi AP, et al. Clinical correlates with anti-MuSK antibodies in generalized seronegative myasthenia gravis. Brain. 2003;126(Pt 10):2304-11.

3. Guptill JT, Sanders DB, Evoli A. Anti-MuSK antibody myasthenia gravis: clinical findings and response to treatment in two large cohorts. Muscle Nerve. 2011;44(1):36-40.

4. Sanders DB, El-Salem K, Massey JM, McConville J, Vincent A. Clinical aspects of MuSK antibody positive seronegative MG. Neurology. 2003;60(12):1978-80.

5. Jaretzki A, Barohn RJ, Ernstoff RM, Kaminski HJ, Keesey JC, Penn AS, et al. Myasthenia gravis: recommendations for clinical research standards. Task Force of the Medical Scientific Advisory Board of the Myasthenia Gravis Foundation of America. Ann Thorac Surg. 2000;70(1):327-34.

6. Barnett C, Bril V, Kapral M, Kulkarni A, Davis AM. Development and validation of the Myasthenia Gravis Impairment Index. Neurology. 2016;87(9):879-86.

7. Padua L, Tonali P, Aprile I, Caliandro P, Bartoccioni E, Evoli A. Seronegative myasthenia gravis: comparison of neurophysiological picture in MuSK+ and MuSK- patients. Eur J Neurol. 2006;13(3):273-6.

8. Prudlo J, Koenig J, Ermert S, Juhász J. Sleep disordered breathing in medically stable patients with myasthenia gravis. Eur J Neurol. 2007;14(3):321-6.

9. Katzberg HD, Vajsar J, Vezina K, Qashqari H, Selvadurai S, Chrestian N, et al. Respiratory Dysfunction and Sleep-Disordered Breathing in Children With Myasthenia Gravis. J Child Neurol. 2020;35(9):600-6.

10. Fernandes Oliveira E, Nacif SR, Alves Pereira N, Fonseca NT, Urbano JJ, Perez EA, et al. Sleep disorders in patients with myasthenia gravis: a systematic review. J Phys Ther Sci. 2015;27(6):2013-8.

11. Heo SJ, Jun JS, Park D, Lee HW, Kim JS, Park JS. Characteristics of obstructive sleep apnea in myasthenia gravis patients: a single center study. Neurol Sci. 2019;40(4):719-24.

12. Fromageot C, Lofaso F, Annane D, Falaize L, Lejaille M, Clair B, et al. Supine fall in lung volumes in the assessment of diaphragmatic weakness in neuromuscular disorders. Arch Phys Med Rehabil. 2001;82(1):123-8.

13. Sarwal A, Walker FO, Cartwright MS. Neuromuscular ultrasound for evaluation of the diaphragm. 


\section{Clinic Stuff}

Muscle Nerve. 2013;47(3):319-29.

14. Nikolić AV, Bačić GG, Daković M, Lavrnić S, Rakočević Stojanović VM, Basta IZ, et al. Myopathy, muscle atrophy and tongue lipid composition in MuSK myasthenia gravis. Acta Neurol Belg. 2015;115(3):361-5.

15. Zouvelou V, Rentzos M, Toulas P, Evdokimidis I. MRI evidence of early muscle atrophy in MuSK positive myasthenia gravis. J Neuroimaging. 2011;21(3):303-5.

16. Niks EH, Kuks JB, Wokke JH, Veldman H, Bakker E, Verschuuren JJ, et al. Pre- and postsynaptic neuromuscular junction abnormalities in musk myasthenia. Muscle Nerve. 2010;42(2):283-8.

17. Martignago S, Fanin M, Albertini E, Pegoraro E, Angelini C. Muscle histopathology in myasthenia gravis with antibodies against MuSK and AChR. Neuropathol Appl Neurobiol. 2009;35(1):103-10.

18. Cenacchi G, Papa V, Valentina P, Fanin M, Marina F, Pegoraro E, et al. Comparison of muscle ultrastructure in myasthenia gravis with anti-MuSK and anti-AChR antibodies. J Neurol. 2011;258(5):746-52.

19. Niks EH, van Leeuwen Y, Leite MI, Dekker FW, Wintzen AR, Wirtz PW, et al. Clinical fluctuations in MuSK myasthenia gravis are related to antigen-specific IgG4 instead of IgGl. J Neuroimmunol. 2008;195(1-2):151-6.

20. Marino M, Basile U, Spagni G, Napodano C, Iorio R, Gulli F, et al. Long-Lasting Rituximab-Induced Reduction of Specific-But Not Total-IgG4 in MuSKPositive Myasthenia Gravis. Front Immunol. 2020;11:613.

21. Iorio R, Damato V, Alboini PE, Evoli A. Efficacy and safety of rituximab for myasthenia gravis: a systematic review and meta-analysis. J Neurol. 2015;262(5):1115-9.

22. Tandan R, Hehir MK, Waheed W, Howard DB. Rituximab treatment of myasthenia gravis: A systematic review. Muscle Nerve. 2017;56(2):185-96.

23. Evoli A, Bianchi MR, Riso R, Minicuci GM, Batocchi AP, Servidei S, et al. Response to therapy in myasthenia gravis with anti-MuSK antibodies. Ann N Y Acad Sci. 2008;1132:76-83.

24. Evoli A, Padua L. Diagnosis and therapy of myasthenia gravis with antibodies to muscle-specific kinase. Autoimmun Rev. 2013;12(9):931-5.

25. Jacob S, Muppidi S, Guidon A, Guptill J, Hehir $\mathrm{M}$, Howard JF, et al. Guidance for the management of myasthenia gravis $(\mathrm{MG})$ and Lambert-Eaton myasthenic syndrome (LEMS) during the COVID-19 pandemic. J Neurol Sci. 2020;412:116803. 